\title{
High-Level Correlated Approach to the Jellium Surface Energy, Without Uniform-Electron-Gas Input
}

\author{
Lucian A. Constantin ${ }^{1}$, J. M. Pitarke ${ }^{2,3}$, J. F. Dobson ${ }^{4}$, A. Garcia-Lekue ${ }^{1}$, and John P. Perdew ${ }^{5}$ \\ ${ }^{1}$ Donostia International Physics Center (DIPC), \\ Manuel de Lardizabal Pasealekua, E-20018 Donostia, Basque Country \\ ${ }^{2}$ CIC nanoGUNE Consolider, Mikeletegi Pasealekua 56, E-2009 Donostia, Basque Country \\ ${ }^{3}$ Materia Kondentsatuaren Fisika Saila, UPV/EHU, and Unidad Física Materiales CSIC-UPV/EHU, \\ 644 Posta kutxatila, E-48080 Bilbo, Basque Country \\ ${ }^{4}$ Nanoscale Science and Technology Centre, Griffith University, Nathan, Queensland 4111, Australia, \\ ${ }^{5}$ Department of Physics and Quantum Theory Group, Tulane University, New Orleans, LA 70118
}

(Dated: November 23, 2007)

\begin{abstract}
We resolve the long-standing controversy over the surface energy of simple metals: Density functional methods that require uniform-electron-gas input agree with each other at many levels of sophistication, but not with high-level correlated calculations like Fermi Hypernetted Chain and Diffusion Monte Carlo (DMC) that predict the uniform-gas correlation energy. Here we apply a very high-level correlated approach, the inhomogeneous Singwi-Tosi-Land-Sjölander (ISTLS) method, and find that the density functionals are indeed reliable (because the surface energy is "bulk-like"). ISTLS values are close to recently-revised DMC values. Our work also vindicates the previouslydisputed use of uniform-gas-based nonlocal kernels in time-dependent density functional theory.
\end{abstract}

PACS numbers: 71.10.Ca,71.15.Mb,71.45.Gm

Density-functional theory (DFT) [1] provides groundstate electron densities and energies (or, in its timedependent version (TDDFT) [2], excitation energies) for atoms, molecules, and solids. Because of its simple selfconsistent-field structure, DFT is used for electronicstructure calculations almost exclusively in condensed matter physics, and heavily in quantum chemistry. Exact in principle, the theory requires in practice approximations for the exchange-correlation (xc) energy (or for the $\mathrm{xc}$ kernel) as a functional of the density. All commonlyused nonempirical approximations require input from the uniform electron gas, which is transferred to inhomogeneous densities. The reliability of these approximations must be judged a posteriori, and there is a long-standing puzzle related to their reliability for solid surface energies, with implications for vacancies and clusters [3].

The surface energy $\sigma$ is the energy cost per unit area to split a bulk solid along a plane. This is not only of technological importance but also a classic and highly sensitive test case for theories of exchange and correlation in many-electron systems. The simplest model is jellium, in which a uniform positive background of density $n=3 / 4 \pi r_{s}^{3}=k_{F}^{3} / 3 \pi^{3}$ terminates sharply at a plane and is neutralized by electrons that penetrate into the vacuum. A local density approximation (LDA) calculation of jellium and simple-metal surface energies [4] showed that the xc component $\sigma^{x c}$ can be several times bigger than the total $\sigma$, and stimulated work [5] that led to the development of more sophisticated functionals.

There is now a ladder of nonempirical semilocal density functionals, with each new rung corresponding to the addition of another ingredient for the energy density. The first rung (LDA) [1] predicts [4, 6] a positive $\sigma^{x c}$ for jellium. The second rung or generalized gradi- ent approximation (GGA) [7] predicts [6] values about $3 \%$ smaller than LDA, while the third rung or metaGGA [8] predicts [6] values about $2 \%$ larger than LDA. Ascent of the ladder brings steady improvement [6] in the exactly-known [9] exchange part $\sigma^{x}$. The randomphase approximation (RPA), which predicts inaccurate correlation energies for the uniform gas, predicts values for $\sigma^{x c}$ about $6 \%$ larger than LDA [9]. The semilocal functionals may be corrected for long-range Coulomb effects [6], and the RPA may be corrected semilocally for short-range correlation [10], producing values 2 or $3 \%$ bigger than LDA. Use, in the framework of TDDFT, of a uniform-gas-based nonlocal xc kernel [11] to correct RPA produces an RPA-like $\sigma^{x c}$, because RPA makes compensating errors for density fluctuations of large and intermediate wavevector [11].

The surface $x c$ energies from all of the above methods disagree strongly with those from existing high-level correlated methods: At $r_{s}=4$ (the bulk density of sodium metal), $\sigma^{x c}$ is about $45 \%$ bigger than its LDA value in the Fermi Hypernetted Chain (FHNC//0) [12] and Diffusion Monte Carlo (DMC) [13] calculations for jellium slabs. One conclusion from this might be that both existing semilocal density functionals (in the framework of DFT) and uniform-gas-based versions of TDDFT are not valid for predicting correlation energies, a very dissappointing outcome that would severely limit the practical usefulness of DFT and TDDFT. In this Letter, we show that this is not the case, and in the process we resolve the controversy over the surface energy of simple metals.

Refs. [11, 14] already showed that a careful analysis of the DMC slab calculations might bring them into agreement with the density functional or RPA values, and also with surface energies extracted from DMC calcu- 
TABLE I: The negative of the correlation energy per electron (in mRyd) of a uniform electron gas in three and two dimensions, where $r_{s}$ is the radius respectively of a sphere or circle containing on average one electron. FHNC//0: Ref. [12]. STLS 3D: Ref. [22]. STLS 2D: Ref. [23]. DMC 3D: Perdew-Wang parametrization of Ceperley-Alder Diffusion Monte Carlo (Ref. [24]). DMC 2D: Parametrization of Eq. (21) of Ref. [25].

\begin{tabular}{lcccc}
\hline \hline dim. & $r_{s}$ & FHNC//0 & STLS & DMC \\
\hline $3 \mathrm{D}$ & 2 & 81.5 & 91.4 & 89.5 \\
& 3 & 65.3 & 74.7 & 73.9 \\
& 4 & 55.0 & 64.0 & 63.7 \\
& 5 & 47.8 & 56.3 & 56.4 \\
& & & & \\
& 1 & & 211 & 219 \\
& 2 & & 155 & 165 \\
& 4 & & 108 & 113 \\
& 8 & & 66 & 72 \\
\hline \hline
\end{tabular}

lations for jellium spheres [15, 16], suggesting that the surface energy puzzle had been solved. Nevertheless, one piece of the puzzle remained. Krotscheck and Kohn [17] examined a collective RPA and used several xc kernels to correct for short-range effects. When they used an isotropic xc kernel derived from the uniform gas, in the spirit of the TDDFT calculation of Ref. [11] (see also Ref. [18]), they found surface energies very close to RPA, as in Ref. [11]; when they used an orbital-based Fermi hypernetted chain approximation $(\mathrm{FHNC} / / 0)$, corresponding to an anisotropic kernel constructed explicitly for the jellium surface, they found a large positive correction to the RPA surface energy. Because of this, they concluded that "The local-density approximation for the particlehole interaction is inadequate to calculate the surface energy of the simple metals".

Here we apply a very high-level correlated approach to calculate $\sigma$, finding values that lie in the narrow range between meta-GGA and RPA, and much lower than the existing DMC or FHNC//0 slab extrapolations. We use an inhomogeneous orbital-based approach (ISTLS) [19] that generalizes the Singwi-Tosi-Land-Sjölander (STLS) formalism [20]. ISTLS is, like the RPA it corrects, a fifthrung density functional that employs the occupied and unoccupied Kohn-Sham orbitals. A comparison between our calculations, which do not use an isotropic xc kernel derived from the uniform gas, and the calculations of Ref. [11] leads us to the conclusion that the LDA for the particle-hole interaction is indeed adequate to describe simple metal surfaces and that existing DFT and RPA surface-energy calculations are reliable.

For the homogeneous electron gas, the STLS approach made a remarkably accurate prediction of the correlation energy, as confirmed by later DMC calculations (see Table I). For an arbitrary inhomogeneous many- electron system, Dobson et al. [19] used the linearity and time-invariance of a truncated Bogoliubov-Born-GreenKirkwood-Yvon (BBGKY) equation [21] to propose what they called an inhomogeneous STLS (ISTLS) scheme, which can be written as a Dyson-like "screening" integral equation for the density-response function:

$$
\chi\left(\mathbf{r}, \mathbf{r}^{\prime} ; \omega\right)=\chi^{0}\left(\mathbf{r}, \mathbf{r}^{\prime} ; \omega\right)+\int d \mathbf{r}^{\prime \prime} Q\left(\mathbf{r}, \mathbf{r}^{\prime \prime} ; \omega\right) \chi\left(\mathbf{r}^{\prime \prime}, \mathbf{r}^{\prime} ; \omega\right),
$$

where

$$
Q\left(\mathbf{r}, \mathbf{r}^{\prime} ; \omega\right)=-\int d \mathbf{r}^{\prime \prime} \boldsymbol{\nu}^{0}\left(\mathbf{r}, \mathbf{r}^{\prime \prime} ; \omega\right) \cdot g\left(\mathbf{r}^{\prime \prime}, \mathbf{r}^{\prime}\right) \nabla_{\mathbf{r}^{\prime \prime}} \frac{1}{\left|\mathbf{r}^{\prime \prime}-\mathbf{r}^{\prime}\right|}
$$

Here, $\boldsymbol{\nu}^{0}\left(\mathbf{r}, \mathbf{r}^{\prime} ; \omega\right)$ is a vector response function defined from the equation $\chi^{0}\left(\mathbf{r}, \mathbf{r}^{\prime} ; \omega\right)=\nabla_{\mathbf{r}^{\prime}} \cdot \boldsymbol{\nu}^{0}\left(\mathbf{r}, \mathbf{r}^{\prime} \omega\right)$, $\chi^{0}\left(\mathbf{r}, \mathbf{r}^{\prime}, \omega\right)$ being the density-response function of noninteracting Kohn-Sham (KS) electrons [26], and the equilibrium pair-correlation function $g\left(\mathbf{r}, \mathbf{r}^{\prime}\right)$ is obtained from the fluctuation-dissipation theorem as follows [5]:

$g\left(\mathbf{r}, \mathbf{r}^{\prime}\right)=1-\frac{1}{\pi n(\mathbf{r}) n\left(\mathbf{r}^{\prime}\right)} \int_{0}^{\infty} d u \chi\left(\mathbf{r}, \mathbf{r}^{\prime} ; i u\right)-\frac{\delta\left(\mathbf{r}-\mathbf{r}^{\prime}\right)}{n\left(\mathbf{r}^{\prime}\right)}$.

Equations (1)-(3) are solved selfconsistently, starting from $\chi^{R P A}\left(\right.$ or $\left.\chi^{0}\right)$, until a converged solution is obtained [27].

By setting $g\left(\mathbf{r}, \mathbf{r}^{\prime}\right)=1$ (the Hartree limit) in Eq. (2) and performing an integration by parts, the RPA is nicely recovered, as expected. In contrast, the inhomogeneous $\mathrm{FHNC} / / 0$ does not recover the actual RPA but a "collective" RPA instead [17]. We note, however, that the ISTLS scheme cannot be written using an xc kernel and does not satisfy the reciprocity constraint, i.e., $\chi^{I S T L S}\left(\mathbf{r}, \mathbf{r}^{\prime} ; \omega\right) \neq \chi^{I S T L S}\left(\mathbf{r}^{\prime}, \mathbf{r} ; \omega\right)$, although for jellium slabs this constraint is found to hold rather well. We also note that ISTLS is exact for all one-electron densities, for which $g\left(\mathbf{r}, \mathbf{r}^{\prime}\right)=0$, and is exact in the high-density limit.

For the evaluation of the ISTLS density-response function, we extend the method described in the Appendix of Ref. [28]. We consider a jellium slab, assume that $n(z)$ vanishes at a distance $z_{0}$ from either jellium edge [29], and expand the single-particle orbitals $\phi_{l}(z)$ and the density-response function $\chi\left(\mathbf{r}, \mathbf{r}^{\prime} ; \omega\right)$ in sine and doublecosine Fourier representations, respectively. Because the integral in Eq. (3) is slowly convergent and must cancel out a space delta function, we use an expression for $g\left(\mathbf{r}, \mathbf{r}^{\prime}\right)$ in terms of the Hartree-Fock pair-correlation function and the density-response functions $\chi^{0}\left(\mathbf{r}, \mathbf{r}^{\prime} ; \omega\right)$ and $\chi\left(\mathbf{r}, \mathbf{r}^{\prime} ; \omega\right)$. We then use the adiabatic-connection fluctuation-dissipation formula $[5,9]$ to obtain the xc surface energy from the following equation:

$$
\sigma^{x c}=\int_{0}^{\infty} d\left(q / k_{F}\right) \gamma_{q}^{x c}
$$

where $q$ represents the magnitude of a wave vector parallel to the surface, and $\gamma_{q}^{x c}$ is given by Eqs. (2) and (3) of 


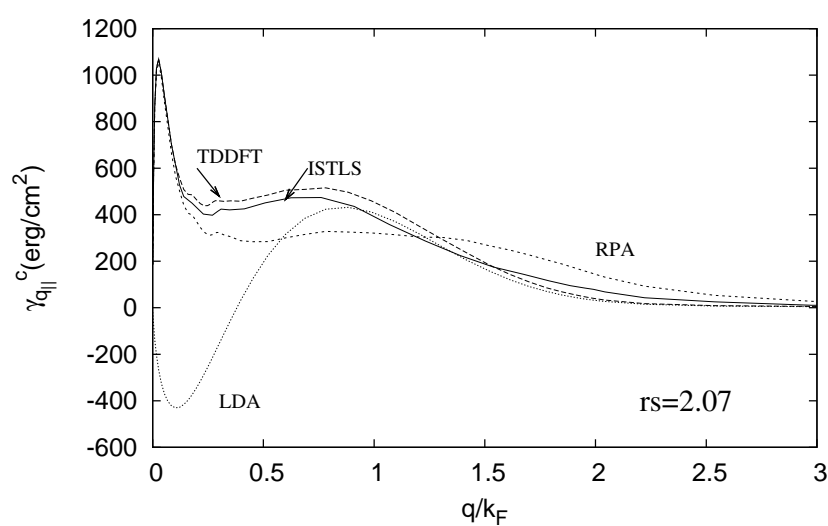

FIG. 1: Wave-vector analysis $\gamma_{q}^{c}$ of the correlation surface energy for a jellium slab of thickness $7.21 r_{s}$ and $r_{s}=2.07$. Solid, thick dashed, thin dashed, and dotted lines represent ISTLS, uniform-gas based TDDFT (as reported in Ref. [11]), RPA, and LDA calculations, respectively. $q$ is the magnitude of the $2 \mathrm{D}$ wavevector (in the surface plane) of the density fluctuations. The area under each curve amounts to the correlation surface energy $\sigma^{c}$. $\left(1\right.$ hartee $/ \mathrm{bohr}^{2}=1.557 \times 10^{6} \mathrm{erg} / \mathrm{cm}^{2}$.)

Ref. [11], the density-response function $\chi_{q, \lambda}\left(z, z^{\prime} ; \omega\right)$ now being the 2D Fourier transform of our ISTLS densityresponse function $\chi_{\lambda}\left(\mathbf{r}, \mathbf{r}^{\prime} ; \omega\right)$ of a fictitious jellium slab of fixed density $n(z)$ at coupling strength $\lambda e^{2}$.

If one replaces the interacting density-response function $\chi_{q, \lambda}\left(z, z^{\prime} ; \omega\right)$ entering Eq. (3) of Ref. [11] by the noninteracting density-response function $\chi_{q}^{0}\left(z, z^{\prime} ; \omega\right)$, then the exact $\sigma^{x}$ is obtained, as in Ref. [9]. Here we focus our attention on $\sigma^{c}$, which for comparison we also calculate (i) in the LDA by replacing $n_{q, \lambda}^{c}$ in Eq. (2) of Ref. [11] by the uniform-gas correlation-hole density at the local density $n(z)$, and (ii) within TDDFT by introducing in Eq. (3) of Ref. [11] the TDDFT density-response function $\chi_{q, \lambda}^{T D D F T}\left(z, z^{\prime} ; \omega\right)$. Within TDDFT, the xc kernel entering Eq. (4) of Ref. [11] is taken to be either zero (RPA) or the uniform-gas based isotropic xc kernel given by Eqs. (6) and (7) of Ref. [11].

Figure 1 shows the wave-vector analysis $\gamma_{q}^{c}$ of our ISTLS correlation surface energy $\sigma^{c}$ (solid line), together with the corresponding wave-vector analysis of (i) the LDA correlation surface energy (dotted line), as obtained by using the Perdew-Wang (PW) parametrization of the uniform-gas correlation-hole density [30], (ii) the RPA correlation surface energy (thin dashed line), and (iii) the isotropic xc-kernel based TDDFT correlation surface energy of Ref. [11] (thick dashed line). We observe that in the long-wavelength limit $(q \rightarrow 0)$ both ISTLS and TDDFT calculations coincide with the RPA, which is exact in this limit, while the LDA fails badly [31]. In the large- $q$ limit, both ISTLS and TDDFT calculations approach the LDA, as expected, while the RPA is
TABLE II: LDA, RPA, ISTLS, TDDFT, TPSS [8], and recent DMC [34] (per Eq. (5)) xc surface energies. Units are $\mathrm{erg} / \mathrm{cm}^{2}$. The numerical grids we use for ISTLS are found to be inadequate even for RPA when $r_{s}>3.28$; nevertheless, our best ISTLS estimates for $r_{s}>3.28$ are found to be very close to the RPA. Values in parentheses represent extrapolations on $r_{s}$ from Eq. (5). Extrapolated values from slab calculations (as here for RPA, ISTLS, TDDFT) typically agree within 1 $\mathrm{erg} / \mathrm{cm}^{2}$ with values from a semi-infinite jellium code (as here for LDA, TPSS).

\begin{tabular}{lcccccc}
\hline \hline$r_{s}$ & $\sigma_{\text {LDA }}^{x c}$ & $\sigma_{\text {RPA }}^{x c}$ & $\sigma_{\text {ISTLS }}^{x c}$ & $\sigma_{\text {TDDFT }}^{x c}$ & $\sigma_{\text {TPSS }}^{x c}$ & $\sigma_{\text {DMC }}^{x c}$ \\
\hline 2.00 & 3357 & 3467 & 3417 & 3466 & 3380 & $(3392 \pm 50)$ \\
2.07 & 2962 & 3064 & 3026 & 3063 & 2983 & $2993 \pm 45$ \\
2.30 & 2019 & 2098 & 2072 & 2096 & 2034 & $2039 \pm 27$ \\
2.66 & 1188 & 1240 & 1227 & 1239 & 1198 & $1197 \pm 13$ \\
3.00 & 764 & 801 & 800 & 797 & 772 & $768 \pm 10$ \\
3.28 & 550 & 579 & 580 & 577 & 557 & $551 \pm 8$ \\
4.00 & 262 & 278 & $(281)$ & 278 & 266 & $(261 \pm 8)$ \\
6.00 & 53.6 & 58 & $(60.5)$ & 58 & 55 & $(53 \pm \ldots)$ \\
\hline \hline
\end{tabular}

wrong [32]. The important lesson that we learn from Fig. 1 is that two independent schemes: (i) our ISTLS approach, which does not use an isotropic kernel derived from the uniform gas, and (ii) the TDDFT approach of Ref. [11], which uses a uniform-gas based isotropic xc kernel, yield essentially the same wave-vector analysis of $\sigma^{c}$. This supports the conclusion that the local-density approximation for the particle-hole interaction is indeed adequate to describe simple metal surfaces.

To extract the surface energy of a semi-infinite medium, we have considered three different values of the slab thickness: the threshold width at which the $n=5$ subband for the $z$ motion is completely occupied and the two widths at which the $n=5$ and $n=6$ subbands are half occupied, and have followed the extrapolation procedure of Ref. [9]. In Table II, we show our extrapolated RPA, TDDFT, and ISTLS xc surface energies, for various values of $r_{s}$. Our ISTLS calculations indicate that a persistent cancellation of short-range xc effects (beyond the RPA) still occurs, as in the case of the uniform-gas based TDDFT calculations of Ref. [11]. However, this cancellation is found not to be as complete as in Ref. [11], and yields xc surface energies that are slightly lower than in the RPA but still a little higher than in the LDA. Indeed, the difference between our ISTLS surface energies and their RPA counterparts is very close to the difference between the conventional GGA [7] surface energies and the corresponding RPA-based GGA surface energies, thereby supporting the assumption made in Ref. [10] that the short-range (beyond RPA) part of the correlation energy can be treated within the GGA. Our ISTLS calculations are also very close to the xc surface energies obtained by using the non-empirical TPSS meta-GGA xc energy functional [8] and a Laplacian-level metal-GGA [33]. 
The FHNC//0 approach yields a large positive correction to the RPA surface energy. However, the FHNC//0 approach used in Ref. [17] is in fact less accurate than STLS for the homogeneous 3D electron gas (see Table I). STLS also does very well for the $2 \mathrm{D}$ electron gas (see Table I). These are reasons to prefer the ISTLS over FHNC//0 for the surface problems we are considering.

The fixed-node DMC calculations of Ref. [13] were critiqued in Refs. [11], [14], and [34]. Recent DMC calculations by Wood et al. [34] suggest that the fixed-node approximation introduces an error that is slightly larger in the slab than in the bulk and indicate that actual DMC surface energies are larger than in the LDA but smaller than in the RPA, as occurs with our ISTLS calculations.

The recent DMC calculations [34] report total surface energies for LDA orbitals (from which $\sigma^{x c}$ is easily extracted) and error bars for $r_{s}=2.07,2.30,2.66,3.25$, and 3.94. To refine, interpolate, and extrapolate these values, we fit to them the physically-motivated form [16]

$$
\sigma^{x c}\left(r_{s}\right)=A /\left[r_{s}^{7 / 2}\left(1+B x+C x^{2}+D x^{3}\right)\right],
$$

where $x=\sqrt{\left(1+r_{s}\right)}-1$. We choose typical values $A=$ $50,000 \mathrm{erg} / \mathrm{cm}^{2}$ (correct $r_{s} \rightarrow 0$ limit) and $D=0.248$ (LDA fit), and then vary $B$ and $C$ to minimize the sum of the squares of the fit deviation divided by the DMC error bar, finding $B=0.6549$ and $C=-0.511$. Note from Table II that LDA and TPSS both lie within the error bars of the recent DMC, while RPA, TDDFT, and ISTLS lie a little higher. The same fit has been made for ISTLS, with $B=0.7437$ and $C=-0.653$.

Finally, we note that an analysis of the origin of the xc surface energy brings us to the conclusion that this quantity is actually "bulk-like", arising mostly from the moderately-varying-density region inside the classical turning plane. (For $r_{s}=2$, only $-3 \%$ of the total $\sigma^{x c}$ comes from the region outside. This increases to $-18 \%$ for $r_{s}=4$.) Inside, the reduced density gradient $s$ falls in a range $(0 \leq s<1.9)$ found in the bulk of real solids, where gradient corrections to LDA exchange and LDA correlation tend to cancel [35].

In summary, we have used a very high-level numerically-expensive correlated approach, the ISTLS method, to analyze the jellium surface energy into contributions from dynamical density fluctuations of various $2 \mathrm{D}$ wave vectors. This analysis rules out the belief that the LDA for the particle-hole interaction might be inadequate to calculate the surface energy of simple metals. Furthermore, our calculations, which are reasonably close to uniform-gas based TDDFT calculations [11] and not far from the LDA, support the old idea that the xc surface energy should be well-described within LDA [5], and resolve the long-standing surface-energy controversy.

L.A.C., J.M.P., and A.G.-L. acknowledge partial support by the Spanish MEC and the EC NANOQUANTA. J.P.P. acknowledges NSF support (Grant No. DMR0501588). We thank H. Le for supplying some data.
[1] W. Kohn and L. J. Sham, Phys. Rev. 140, A1133 (1965).

[2] E. Runge and E. K. U. Gross, Phys. Rev. Lett. 52, 997 (1984); M. Mundt and S. Kümmel, Phys. Rev. Lett. 95, 203004 (2005).

[3] J. P. Perdew et al., Phys. Rev. Lett. 66, 508 (1991).

[4] N. Lang and W. Kohn, Phys. Rev. B 1, 4555 (1970).

[5] D. C. Langreth and J. P. Perdew, Phys. Rev. B 15, 2884 (1977).

[6] L. A. Constantin et al., Phys. Rev. B 73, 205104 (2006).

[7] J. P. Perdew et al., Phys. Rev. Lett. 77, 3865 (1996).

[8] J. Tao et al., Phys. Rev. Lett. 91, 146401 (2003).

[9] J. M. Pitarke and A. G. Eguiluz, Phys. Rev. B 57, 6329 (1998); 63, 045116 (2001).

[10] Z. Yan et al., Phys. Rev. B 61, 16430 (2000).

[11] J. M. Pitarke and J. P. Perdew, Phys. Rev. B 67, 045101 (2003).

[12] E. Krotscheck et al., Phys. Rev. B 32, 5693 (1985).

[13] P. H. Acioli and D. M. Ceperley, Phys. Rev. B 57, 17199 (1996).

[14] J. M. Pitarke, Phys. Rev. B 70, 087401 (2004).

[15] F. Sottile and P. Ballone, Phys. Rev. B 64, 045105 (2001).

[16] L. M. Almeida et al., Phys. Rev. B 66, 75115 (2002).

[17] E. Krotscheck and W. Kohn, Phys. Rev. Lett. 57, 862 (1986).

[18] J. Jung et al., Phys. Rev. B 64, 205107 (2004).

[19] J. F. Dobson et al., Phys. Rev. B 66, 081108 (2002).

[20] K. S. Singwi et al., Phys. Rev. 176, 589 (1968).

[21] T. J. M. Boyd and J. J. Sanderson, Plasma Dynamics (Barnes and Noble, New York, 1969).

[22] J. Dobson, H. Le, and J. Wang, (unpublished).

[23] M. Jonson, J. Phys. C: Solid State Phys. 9, 3055 (1976).

[24] J. P. Perdew and Y. Wang, Phys. Rev. B 45, 13244 (1992); D. M. Ceperley and B. J. Alder, Phys. Rev. Lett. 45, 566 (1980).

[25] Y. Kwon et al., Phys. Rev. B 48, 12037 (1993).

[26] $\chi^{0}\left(\mathbf{r}, \mathbf{r}^{\prime} ; \omega\right)$ should be determined from the exact $\mathrm{KS}$ eigenfunctions and eigenvalues. The error introduced by our use of their LDA counterparts, as obtained by using the PW parametrization [24], is expected to be small.

[27] Four or five cycles are found to yield a converged solution.

[28] A. G. Eguiluz, Phys. Rev. B 31, 3303 (1985).

[29] $z_{0}$ is chosen sufficiently large for the physical results to be insensitive to the precise value employed.

[30] J. P. Perdew and Y. Wang, Phys. Rev. B 46, 12947 (1992).

[31] The LDA is known to be a much better approximation for $\gamma_{q}^{x c}$ than for $\gamma_{q}^{c}$ alone, even at long wavelengths $(q \rightarrow 0)$.

[32] In the large- $q$ limit, the RPA (ISTLS) reproduces an RPA-based (STLS-based) LDA obtained from the RPA (STLS) uniform-gas correlation-hole density. Differences at large $q$ between the RPA (ISTLS) and LDA calculations shown in Fig. 1 are the result of differences between the RPA (STLS) uniform-gas correlation-hole density and the more accurate PW parametrization, which in the case of the RPA are considerably large.

[33] J. P. Perdew and L. A. Constantin, Phys. Rev. B, 75, 155109 (2007).

[34] B. Wood et al., Phys. Rev. B 76, 035403 (2007).

[35] Continuity arguments suggest that, not far outside, the LDA should still be a reasonable approximation. 